little evidence available regarding NIV compliance and the factors that affect this. Accurate compliance data from individual ventilators is readily available and gives a new opportunity to explore this further. The aim of this research was to describe and analyse adherence with NIV among established patients and determine if this was significantly affected by age, gender, diagnosis, time since commencement, apnoea-hypopnoea index (AHI), mean daily usage, leak or pressure.

Methods Ventilator download-data from sleep study databases and electronic patient-notes were retrospectively reviewed. Patients aged 0-18, established on NIV with between 30-182 days of available data were included. Adherence, defined as percentage of nights with $\geq 4 \mathrm{~h}$ use, was compared by diagnosis, gender and age and correlated with the above factors.

Results 102 children were included, 67\% were male and median age was 13 years (range $0.25-18$ ). The mean adherence for all patients was $64.2 \%$. There was a strong positive correlation between mean daily usage and compliance $(r=0.74)$. Children with Down's syndrome had a mean adherence of $37.9 \%$, considerably lower than in other diagnoses including obstructive sleep apnoea (62.3\%), craniofacial conditions $(61.7 \%)$, neuromuscular disease $(64.7 \%)$ and conditions with central hypopnoeas (80.5\%). Gender and presence of leak did not affect adherence and no correlation between adherence and ventilator pressure $(\mathrm{r}=0.30)$, age $(\mathrm{r}=0.05)$ or AHI $(\mathrm{r}=0.08)$ was demonstrated.

Conclusion It is clear from the few correlations found that patients are heterogeneous and the challenges multifactorial. A better understanding of adherence levels in individual patients can now enable us to have open discussions with families to address challenges they face with the use of NIV. Children with Down's syndrome are normally carefully selected prior to establishment of NIV because of known difficulties with treatment. Despite this, they are still the group with poorest adherence, highlighting the need for novel approaches to improve this. Ongoing use of NIV does suggest that although adherence is far from perfect, patients must gain some clinical benefit and the levels of adherence truly required to reduce long term complications is still not known.

\section{G380(P) HYPONATREMIA - CEREBRAL SALT WASTING}

RN Anantharaman. Paediatric Intensive Care Unit (PICU), Oxford University Hospital NHS Trust, Oxford, UK

\subsection{6/archdischild-2015-308599.335}

\section{Setting PICU}

Consultant and PICU registrar

Problem 4 year old child transferred to PICU after space occupying lesion cranial surgery. On day 2 post operatively was noted to have very high urine output with falling serum sodium. His urine output was very variable and ranged from $3 \mathrm{ml} / \mathrm{kg} /$ hour to $17 \mathrm{ml} / \mathrm{kg} /$ hour. He had very high urinary sodium content.

Clinical context

- Falling sodium

- High urine output

- Low plasma osmolality

Assessment of problem and analysis of its causes

- Hyponatremia in Patients with Neurologic Disorders

- Inappropriate secretion of antidiuretic hormone (SIADH)

- Cerebral salt wasting (CSW) syndrome
The distinction between these two conditions is important because their treatments are different. It is not possible to distinguish CSW from SIADH based on serum and urine laboratory findings alone, because their associated abnormalities are identical. For this reason, accurate determination of the patient's volume status is the key to differentiating these syndromes.

Fundamental difference between the two processes is extracellular fluid volume status. Patients with CSW have hypovolemia compared with patients with SIADH, who have euvolemic or mild extra cellular fluid expansion.

The mainstay of therapy for CSW is replacement of the sodium and water that is lost as a result of pathologic natriuresis and diuresis. This is in direct contrast to the treatment of SIADH, the crux of which is free water restriction.

CVP-directed treatment of hyponatremia and volume status in such patients is effective.

Intervention New guidelines for cerebral salt wasting introduced in PICU guidelines.

Lessons learnt Consider CSW in children with low sodium and high urine output, measure volume status, in PICU setting with CVP measurement

\section{G381(P) IMMUNOPROTECTIVE EFFECTS OF INCHALED NITRIC OXIDE IN NEWBORNS WITH RESPIRATORY DISEASES ON MECHANICALLY VENTILATION}

M Puhtinskaya, V Estrin. Department of Anesthesiology and Intensive Care, Research Institute of Obstetrics and Pediatrics, Rostov-on-Don, Russia

\subsection{6/archdischild-2015-308599.336}

Introduction This study presents the results of the influence of inhaled nitric oxide (iNO) on the immune system of infants with respiratory diseases on mechanically ventilation (MV).

Purpose Decrease neonatal mortality.

Materials and methods In a controlled, randomised, blinded clinical trial 37 newborns with respiratory diseases on MV were included. In group I 20 patients received iNO (10 ppm, $24 \mathrm{~h}$; "Pulmonox mini", "Messer II NO Therapeutics", Austria). Newborns in group II $(n=17)$ did not receive iNO. On admission and at 3-5 days, subpopulations of lymphocytes were studied by the one-parameter immunophenotyping using reagents company Immunotech Beckman Coulter (USA): CD3, CD4, CD8, CD14, CD19, CD34, CD56, CD69, CD71, CD95 monoclonal antibody, the relative content of lymphocytes in apoptosis using AnneksinV + labelled FITK and propidium iodide (PL+), labelled with PE (Saltag, USA). The statistical power of the study was $80 \%(\alpha \leq 0.05)$.

Results In group I an increase of mature monocytes $\left(\mathrm{CD} 14^{+}\right)$to $23.1 \pm 0.8 \%(\mathrm{p}<0.05)$ was observed on $3-5$ days. The relative content of CD69 was reduced to $3.8 \pm 0.21 \%$, and in lymphocytes in apoptosis to $7.12 \pm 0.46 \%$ (AnnexinV-FITC ${ }^{+} \mathrm{PI}$ ), and to $0.79 \pm 0.07 \%$ (AnnexinV-FITC ${ }^{+} \mathrm{PI}^{+}, \mathrm{p}<0.001$ ). Duration of $\mathrm{MV}$ was $4.1 \pm 1.4$ days in group I and $18 \pm 3.4$ in group II. All newborns in group I survived and had no septic complications. None of the patients showed clinical or laboratory evidence of adverse effects of iNO. In group II 7 newborns died, 5 developed neonatal sepsis.

Conclusion Inhaled NO in mechanically ventilated newborns increased the relative content of mature macrophages and decreased the number of lymphocytes in apoptosis. Most importantly, the incidence of sepsis and fatal outcome was reduced in iNO-treated newborns. 